\title{
Cotilting objects and dualities
}

\author{
Robert Wisbauer \\ Mathematical Institute of the University \\ 40225 Düsseldorf, Germany
}

\begin{abstract}
Tilting modules generalize projective generators and may be characterized either by weakened generating and projectivity conditions or else by equivalences they define between certain subcategories. Dually cotilting modules generalize injective cogenerators and there are again principally two ways to describe them: first by weakened cogenerating and injectivity conditions, and second by dualities they induce between suitable subcategories. In this paper we begin with several characterizations related to the first point of view, and it turns out that for properties of the second type certain finiteness conditions are needed - similar to the situation for Morita dualities for rings.
\end{abstract}

\section{Introduction}

Dualizing tilting modules, cotilting modules $Q$ in $R$-Mod are defined in [7] by the conditions

(1) $\operatorname{inj} \operatorname{dim}\left({ }_{R} Q\right) \leq 1$,

(2) $\operatorname{Ext}_{R}^{1}\left(Q^{\Lambda}, Q\right)=0$, for any set $\Lambda$,

(3) for all $N \in R$-Mod, $\operatorname{Hom}_{R}(N, Q)=0=\operatorname{Ext}_{R}^{1}(N, Q)$ implies $N=0$.

In Section 1 various injectivity and cogenerating conditions are introduced for objects (in Grothendieck categories), which result from dualizing notions of interest in the study of (self-) tilting objects. Self-tilting modules $M$ are those which are tilting in the category $\sigma[M]$, whose objects are submodules of $M$-generated modules, and they are precisely the *-modules (introduced by Menini-Orsatti, see [16]). For the characterization of (self-) cotilting modules we introduce the category $\pi[M]$, whose objects are factor modules of $M$-cogenerated modules. In Section 3 cotilting modules in $\pi[M]$ and $R$-Mod are introduced by injectivity and cogenerating conditions and it is shown in 3.5 that these modules coincide with those mentioned above. 
Over artinian rings the two categories $\sigma[M]$ and $\pi[M]$ coincide and this is one of the reasons why in representation theory of finite-dimensional algebras tilting and cotilting modules are so closely connected by formal duality.

The interest in tilting modules arose from the fact that they provide equivalences between certain categories. So one expects certain dualities for the dual notion, the cotilting modules. However it is well known that there are no dualities between full module categories and one has to restrict to finitely closed subcategories. We will see in 4.8 and 4.10 that under some finiteness conditions cotilting modules yield such dualities.

Our techniques and results subsume and generalize previous work on the subject by Colby [4], Wang-Xu [13], Angeleri-Hügel, Colpi, Fuller, Tonolo, Trlifaj [8, 3, 5, 6], and others. For the special case of a faithfully balanced bimodule over an artinian algebra related results are obtained in Zhaoyong [18].

\section{Preliminaries}

Throughout the paper $\mathcal{C}$ will denote a locally finite Grothendieck category, i.e., a cocomplete abelian category with exact direct limits and a generating set of finitely generated objects (e.g., [11, Chapter V]). Moreover $R$ will be an associative ring with unit, $R$-Mod the category of unital left $R$-modules and $R$-mod the full subcategory of finitely generated $R$-modules.

1.1 Trace and reject. For any family $\mathcal{X}$ of objects in $\mathcal{C}$ and $N \in \mathcal{C}$, the trace of $\mathcal{X}$ in $N$ is defined by

$$
\operatorname{Tr}(\mathcal{X}, N)=\sum\left\{\operatorname{Im}(f) \mid f \in \operatorname{Hom}_{\mathcal{C}}(X, N), X \in \mathcal{X}\right\} \subset N,
$$

and the reject of $\mathcal{X}$ in $N$ is given by

$$
\operatorname{Re}(N, \mathcal{X})=\bigcap\left\{\operatorname{Ke}(f) \mid f \in \operatorname{Hom}_{\mathcal{C}}(N, X), X \in \mathcal{X}\right\} \subset N .
$$

For $\mathcal{X}=\{X\}$ we simply write $\operatorname{Tr}(X, N)$ and $\operatorname{Re}(N, X)$, respectively.

$1.2 P$-generated and $P$-presented objects. Let $P \in \mathcal{C}$. An object $N \in \mathcal{C}$ is (finitely) $P$-generated if there is an epimorphism $P^{(\Lambda)} \rightarrow N$ (with $\Lambda$ finite), and $N$ is $P$-presented if there exists an exact sequence

$$
P^{\left(\Lambda^{\prime}\right)} \rightarrow P^{(\Lambda)} \rightarrow N \rightarrow 0, \quad \Lambda^{\prime}, \Lambda \text { some sets. }
$$

We write $\operatorname{Gen}(P)$ and $\operatorname{Pres}(P)$ for the full subcategories of $\mathcal{C}$ consisting of $P$-generated, resp., $P$-presented modules. 
1.3 The category $\sigma[M]$. For any object $M \in \mathcal{C}, \sigma[M]$ denotes the full subcategory of $\mathcal{C}$ whose objects are subobjects of $M$-generated objects. $\sigma[M]$ is again a locally finite Grothendieck category and the trace functor

$$
\mathcal{T}^{M}: \mathcal{C} \rightarrow \sigma[M], \quad N \mapsto \mathcal{T}^{M}(N):=\operatorname{Tr}(\sigma[M], N)
$$

is right adjoint to the inclusion functor $\sigma[M] \rightarrow \mathcal{C}$, i.e., $\sigma[M]$ is a coreflective subcategory of $\mathcal{C}$ (e.g., $[14,45.11])$. Notice that for any injective $N \in \mathcal{C}, \mathcal{T}^{M}(N)=\operatorname{Tr}(M, N)$ is an injective object in $\mathcal{C}$.

For $P \in \mathcal{C}, \operatorname{Add}(P)$ (resp. add $(P)$ ) stands for the class of modules which are direct summands of (finite) direct sums of copies of $P$, and obviously

$$
\operatorname{add}(P) \subset \operatorname{Add}(P) \subset \operatorname{Pres}(P) \subset \operatorname{Gen}(P) \subset \sigma[P] \subset \mathcal{C},
$$

where all these inclusions may be proper.

1.4 Tilting objects. An object $P \in \mathcal{C}$ is called tilting in $\mathcal{C}$ if $P$ is $\operatorname{Gen}(P)$-projective, $\operatorname{Gen}(P)=\operatorname{Pres}(P)$, and $P$ is a subgenerator in $\mathcal{C}$. $P$ is said to be self-tilting if it is tilting in the category $\sigma[P]$. Self-tilting modules define an equivalence between $\operatorname{Pres}(P)$ and a suitable subcategory of $\operatorname{End}(P)$-Mod (see [16]).

Now we consider notions which are dual to those presented above.

$1.5 Q$-cogenerated and $Q$-copresented objects. Let $N, Q \in \mathcal{C}$. Then $N$ is (finitely) $Q$-cogenerated if there exists an embedding $N \rightarrow Q^{\Lambda}$ (with $\Lambda$ finite), and $N$ is (finitely) $Q$-copresented if there exists an exact sequence

$$
\left.0 \rightarrow N \rightarrow Q^{\Lambda} \rightarrow Q^{\Lambda^{\prime}}, \quad \Lambda, \Lambda^{\prime} \text { some sets ( } \Lambda \text { finite }\right) .
$$

We write $\operatorname{Cog}(Q)$ and $\operatorname{Cop}(Q)$ for the full subcategories of $\mathcal{C}$ consisting of $Q$ cogenerated, resp., $Q$-copresented modules, and $\operatorname{cop}(Q)$ for the modules which are finitely copresented by $Q$ (notice that for this - in the defining sequence - we do not require $\Lambda^{\prime}$ to be finite). Prod $(Q)$ stands for the direct summands of arbitrary products of $Q$ in $\mathcal{C}$.

Notice that the notions $\operatorname{Cog}(Q), \operatorname{Cop}(Q)$ and $\operatorname{Prod}(Q)$ depend on the category $\mathcal{C}$. If it is necessary we will stress this by writing $\operatorname{Cog}_{\mathcal{C}}(Q), \operatorname{Cop}_{\mathcal{C}}(Q)$, and $\operatorname{Prod}_{\mathcal{C}}(Q)$ for clarity.

1.6 The category $\pi[M]$. For any object $M \in \mathcal{C}$, we denote by $\pi[M]$ the full subcategory of $\mathcal{C}$ whose objects are factor objects of $M$-cogenerated objects. By definition we have

$$
\operatorname{add}(M) \subset \operatorname{Prod}(M) \subset \operatorname{Cop}(M) \subset \operatorname{Cog}(M) \subset \pi[M] \subset \mathcal{C}
$$


It is easy to see that $\sigma[M] \subset \pi[M]$ and $\pi[M]$ is also closed under direct sums, factor objects and subobjects and hence is a (locally finite) Grothendieck subcategory of $\mathcal{C}$. In fact:

For any generator $G$ in $\mathcal{C}$ and $\Lambda:=\operatorname{Hom}_{\mathcal{C}}(G, M)$, we have

$$
\pi[M]=\sigma\left[M^{\Lambda}\right]=\operatorname{Gen}(G / \operatorname{Re}(G, M)) .
$$

Proof. By the canonical monomorphism $G / \operatorname{Re}(G, M) \rightarrow M^{\Lambda}$, we obviously have

$$
\operatorname{Gen}(G / \operatorname{Re}(G, M)) \subset \sigma\left[M^{\Lambda}\right] \subset \pi[M] .
$$

For any $N \in \operatorname{Cog}(M)$, there exists an epimorphism $G^{(\Omega)} \rightarrow N$ which clearly yields an epimorphism $G / \operatorname{Re}(G, M)^{(\Omega)} \rightarrow N$. This implies $N \in \operatorname{Gen}(G / \operatorname{Re}(G, M))$ and $\operatorname{Gen}(G / \operatorname{Re}(G, M))=\pi[M]$.

By the above equalities we know that $\pi[M]$ is a coreflective subcategory of $\mathcal{C}$ (see 1.3). For special categories $\mathcal{C}$ it is also a reflective subcategory:

Assume that in $\mathcal{C}$ products of epimorphisms are epimorphisms. Then

(1) $\pi[M]$ is closed under products in $\mathcal{C}$.

(2) The functor $\mathcal{C} \rightarrow \pi[M], \quad N \mapsto N / \operatorname{Re}(N, \pi[M])$, is left adjoint to the inclusion functor $I: \pi[M] \rightarrow \mathcal{C}$.

(3) For any projective object (generator) $P \in \mathcal{C}, P / \operatorname{Re}(P, \pi[M])$ is a projective object (generator) in $\pi[M]$.

Proof. (1) Consider any family $\left\{N_{\lambda}\right\}_{\Lambda}$ of objects in $\pi[M]$. Then each $N_{\lambda}$ is an image of some $U_{\lambda} \subset M^{\Omega_{\lambda}}$. By assumption, $\prod_{\Lambda} N_{\lambda}$ is a factor module of $\prod_{\Lambda} U_{\lambda}$ and hence belongs to $\pi[M]$.

(2) By (1) the functor is well defined and obviously

$$
\operatorname{Hom}_{\mathcal{C}}(N, I(K)) \simeq \operatorname{Hom}_{\mathcal{C}}(N / \operatorname{Re}(N, \pi[M]), K) \text {, for any } N \in \mathcal{C}, K \in \pi[M] .
$$

(3) This is easily verified.

1.7 AB4* categories. Abelian categories with products in which products of epimorphisms are epimorphisms are called $A B 4^{*}$ categories. It is well known that for any associative ring $R, R$-Mod has this property (e.g., $[14,9.3]$ ). In view of the relationship between the product in $R$-Mod and its coreflective subcategories (see 1.3) it is straightforward to prove:

Assume that for $M \in R$-Mod the trace functor $\mathcal{T}^{M}$ is exact. Then in $\sigma[M]$ products of epimorphisms are epimorphisms.

For characterizations of $\mathcal{T}^{M}$ being exact we refer to $[15,4.6]$. 
Recall that $M \in \mathcal{C}$ is a subgenerator in $\mathcal{C}$, provided $\sigma[M]=\mathcal{C}$. We call $M$ a weak subgenerator in $\mathcal{C}$, provided $\pi[M]=\mathcal{C}$.

1.8 Weak subgenerators. For $M \in \mathcal{C}$ the following conditions are equivalent:

(a) $M$ is a weak subgenerator in $\mathcal{C}$;

(b) for some $\Lambda, M^{\Lambda}$ is a subgenerator in $\mathcal{C}$;

(c) every object in $\mathcal{C}$ is a subfactor of some object in $\operatorname{Cog}(M)$;

(d) every injective object in $\mathcal{C}$ is a factor of some object in $\operatorname{Cog}(M)$;

(e) $\operatorname{Cog}(M)$ contains a generator (a generating set of objects) of $\mathcal{C}$;

(f) $\operatorname{Cog}(M)$ contains a subgenerator of $\mathcal{C}$.

Proof. This can be easily shown by standard arguments.

Clearly any cogenerator of $\mathcal{C}$ is a weak subgenerator but not necessarily a subgenerator of $\mathcal{C}$. For example, $\mathscr{Q} / \mathbb{Z}$ is a cogenerator but is not a subgenerator in $\mathbb{Z}$-Mod $(\sigma[Q \mathbb{Q} / \mathbb{Z}]$ are just the torsion $\mathbb{Z}$-modules).

The following observations are obvious consequences.

\subsection{Corollary.}

(1) If $M$ is a weak subgenerator in $\mathcal{C}$, then $\operatorname{Cog}(M)$ contains all projectives of $\mathcal{C}$.

(2) Assume $\mathcal{C}$ has a (sub-) generating set of finitely cogenerated projective objects. Then $Q$ is a weak subgenerator if and only if $Q$ is a subgenerator in $\mathcal{C}$.

For any $R$-module $M, \pi[M]$ has a particularly nice form:

$1.10 \pi[M]$ in $R$-Mod.

(1) For any $M \in \mathrm{R}$-Mod, $\pi[M]=R / \operatorname{An}(M)$-Mod.

(2) An $R$-module $Q$ is a weak subgenerator in $R$-Mod if and only if $Q$ is a faithful $R$-module.

(3) If ${ }_{R} R$ is finitely cogenerated then every weak subgenerator is a subgenerator in $R$-Mod.

(4) $R$ is left artinian if and only if, for any $R$-module $M, \sigma[M]=\pi[M]$.

In studying dualities the following finitely closed subgategory turned out to be of importance (e.g., $[14,47.12])$. Here it will also help to relate cotilting modules to dualities. 
1.11 The category $\sigma_{f}[M]$. For any object $M \in \mathcal{C}$, let $\sigma_{f}[M]$ denote the full subcategory of $\mathcal{C}$ whose objects are subobjects of finitely $M$-generated objects. This is a finitely closed abelian subcategory of $\sigma[M]$ (see $[14,47.2])$.

For $Q \in \mathcal{C}$, we denote by $\operatorname{Cog}_{f}(Q)$ (resp., $\operatorname{cog}(Q)$ ) the class of (finitely) $Q$ cogenerated objects in $\sigma_{f}[Q]$. Clearly $\operatorname{Cog}_{f}(Q)=\operatorname{Cog}(Q) \cap \sigma_{f}[Q]$, where $\operatorname{Cog}(Q)$ is defined in $\mathcal{C} \cdot \operatorname{cog}(Q)$ is the same when formed in $\sigma_{f}[Q]$ or $\mathcal{C}$ and this also applies to $\operatorname{cop}(Q)$, the class of finitely $Q$-copresented modules (see 1.5).

1.12 Ext-functor in $\mathcal{C}$. By $\operatorname{Ext}_{\mathcal{C}}^{1}$ and $\operatorname{Ext}_{\mathcal{C}}^{2}$ we denote the first and second Extfunctor in $\mathcal{C}$. For $Q \in \mathcal{C}$ and any exact sequence $0 \rightarrow K \rightarrow L \rightarrow N \rightarrow 0$ in $\mathcal{C}$, we have the long exact sequence

$$
\begin{aligned}
0 \rightarrow & \operatorname{Hom}_{R}(N, Q) \rightarrow \operatorname{Hom}_{R}(L, Q) \rightarrow \operatorname{Hom}_{R}(K, Q) \rightarrow \\
& \operatorname{Ext}_{\mathcal{C}}^{1}(N, Q) \rightarrow \operatorname{Ext}_{\mathcal{C}}^{1}(L, Q) \rightarrow \operatorname{Ext}_{\mathcal{C}}^{1}(K, Q) \rightarrow \operatorname{Ext}_{\mathcal{C}}^{2}(N, Q) \rightarrow \cdots .
\end{aligned}
$$

and we denote the kernel of $\operatorname{Ext}_{\mathcal{C}}^{1}(-, Q)$ by

$$
{ }^{\perp_{\mathcal{C}}} Q:=\left\{N \in \mathcal{C} \mid \operatorname{Ext}_{\mathcal{C}}^{1}(N, Q)=0\right\} .
$$

Applied to the Grothendieck category $\pi[M]$, for any $M \in \mathcal{C}$, we have the functors $\operatorname{Ext}_{\pi[M]}^{1}$ and $\operatorname{Ext}_{\pi[M]}^{2}$ and, for $Q \in \pi[M]$, we write

$$
{ }^{\perp_{M}} Q:=\left\{N \in \pi[M] \mid \operatorname{Ext}_{\pi[M]}^{1}(N, Q)=0\right\} .
$$

In particular, for $Q \in \mathcal{C}$ we use the notation

$$
{ }^{\perp_{f}} Q:=\left\{N \in \sigma_{f}[Q] \mid \operatorname{Ext}_{\sigma_{f}[Q]}^{1}(N, Q)=0\right\} .
$$

For $\mathcal{C}=R$-Mod and $M=R$ we apply the usual notation $\operatorname{Ext}_{R}^{1}$ and $\operatorname{Ext}_{R}^{2}$.

\section{Injectivity conditions}

Dualizing projectivity conditions which came up in the study of tilting modules we recall and introduce injectivity properties which are of interest for our further investigations.

2.1 Definitions. Consider exact sequences $(*) \quad 0 \rightarrow K \rightarrow L \rightarrow N \rightarrow 0$ in $\mathcal{C}$.

An object $Q \in \mathcal{C}$ is called

$$
\mathcal{C} \text {-injective }
$$

self-Ext-injective

$\operatorname{Cog}(Q)$-injective

$\operatorname{cog}(Q)$-injective

$w$-П-quasi-injective

$w$ - $\Pi_{f}$-quasi-injective

self-pseudo-injective if $\operatorname{Hom}_{\mathcal{C}}(-, Q)$ is exact on $(*)$

for all sequences $(*)$ in $\mathcal{C}$;

provided $N \in \operatorname{Cog}(Q)$;

provided $L, N \in \operatorname{Cog}(Q)$;

provided $L \in \operatorname{cog}(Q), N \in \operatorname{Cog}(Q)$;

provided $L=Q^{\Lambda}, N \in \operatorname{Cog}(Q)$;

provided $L=Q^{k}, k \in \mathbb{N}, N \in \operatorname{Cog}(Q)$;

provided $K=\operatorname{Re}(L, Q)$. 
The notation $\mathrm{w}-\Pi$-quasi-injective should indicate that this notion is dual to $\mathrm{w}-\Sigma$ quasi-projective. Notice that for the definition of $\operatorname{cog}(Q)$-injective the object $N$ in the sequence $(*)$ is not required to be in $\operatorname{cog}(Q)$ but only in $\operatorname{Cog}(Q)$. We have the obvious implications

$$
\begin{aligned}
& \mathcal{C} \text {-injective } \\
& \Downarrow \\
& \text { self-Ext-injective } \Rightarrow \operatorname{Cog}(Q) \text {-injective } \Rightarrow \text { w-П-quasi-injective } \\
& \Downarrow \quad \Downarrow \quad \Downarrow \\
& \text { self-pseudo-injective } \quad \operatorname{cog}(Q) \text {-injective } \Rightarrow \mathrm{w} \text { - } \prod_{f} \text {-quasi-injective }
\end{aligned}
$$

Notice that in general these implications cannot be reversed. For example, any left hereditary Artin algebra $A$ is self-Ext-injective but not necessarily injective in $A$-Mod; for a non-semisimple ring $R$, any cogenerator $Q$ in $R$-Mod is trivially selfpseudo-injective but need not be self-Ext-injective; for such rings every semisimple $R$-module is $\operatorname{cog}(Q)$-injective ( $\mathrm{w}-\Pi_{f}$-quasi-injective) but not necessarily $\operatorname{Cog}(Q)$ injective (w-П-quasi-injective). We will see in 2.3 that for a module $Q$, w- $\Pi$-quasiinjectivity is equivalent to $\operatorname{Cog}(Q)$-injectivity, provided $\operatorname{Cog}(Q)=\operatorname{Cop}(Q)$.

Next we give some characterizations and properties resulting from this notions.

\subsection{Self-pseudo-injective modules in $\mathcal{C}$.}

(1) For $Q \in \mathcal{C}$ the following are equivalent:

(a) $Q$ is self-pseudo-injective;

(b) for any $N \in \mathcal{C}, \operatorname{Hom}_{\mathcal{C}}(\operatorname{Re}(N, Q), Q)=0$;

(c) $\operatorname{Cog}(Q)$ is closed under extensions in $\mathcal{C}$;

(d) any diagram with exact row in $\mathcal{C}$,

$$
\begin{aligned}
& 0 \rightarrow K \rightarrow L \rightarrow N \rightarrow 0 \\
& \downarrow \quad \beta \dot{v} \\
& Q \quad \stackrel{\alpha}{Q} \quad Q \quad,
\end{aligned}
$$

where $N \in \operatorname{Cog}(Q)$, can be non-trivially commutatively extended by some $\alpha: Q \rightarrow Q, \beta: L \rightarrow Q$.

(2) If $Q$ is self-pseudo-injective then $\operatorname{Cog}(Q)$ is closed under kernels.

Proof. (1) These properties are known or easily verified $([12,2.2],[15,6.5])$.

(2) This is proved in [9, Proposition 4.4], (d) $\Rightarrow(\mathrm{e})$.

Dual to the properties of $\mathrm{w}-\Sigma$-quasi-projective-modules given in $[16,3.2]$ we have the following observations on $\mathrm{w}$-П-quasi-injective objects. 


\section{$2.3 \mathrm{w}$-П-quasi-injective objects.}

(1) For any $Q \in \mathcal{C}$ the following are equivalent:

(a) $Q$ is w-П-quasi-injective;

(b) $\operatorname{Hom}_{\mathcal{C}}(-, Q)$ respects exact sequences $0 \rightarrow K \rightarrow L \rightarrow N \rightarrow 0$, where $N \in \operatorname{Cog}(Q)$ and $L \in \operatorname{Cop}(Q)$.

(2) If $Q$ is w-П-quasi-injective then $\operatorname{Cop}(Q)$ is closed under kernels.

(3) If $\operatorname{Cog}(Q)=\operatorname{Cop}(Q)$ the following are equivalent:

(a) $Q$ is w-П-quasi-injective;

(b) $Q$ is $\operatorname{Cog}(Q)$-injective.

Proof. (1) (b) $\Rightarrow($ a) is trivial.

(a) $\Rightarrow$ (b) For any morphism $h: K \rightarrow Q$, we have a commutative diagram with exact row and columns,

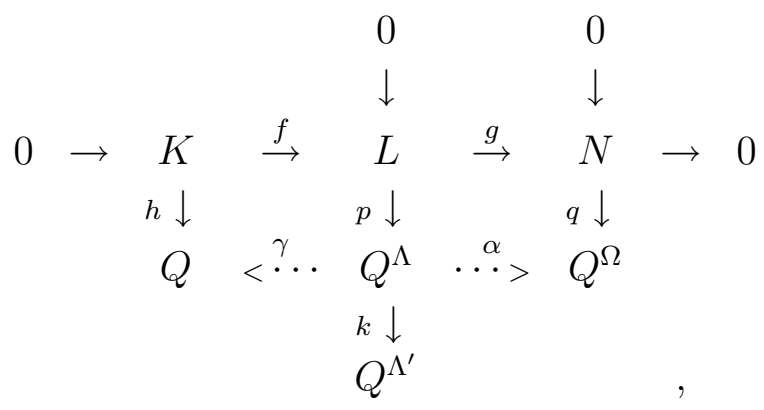

where $\Lambda, \Lambda^{\prime}$, and $\Omega$ are suitable sets. By $(a)$, there exists $\alpha: Q^{\Lambda} \rightarrow Q^{\Omega}$ with $p \alpha=g q$. It is easy to verify that (up to isomorphism) $K f p=\operatorname{Ke} k \cap \operatorname{Ke} \alpha$ and hence $Q^{\Lambda} / K f p \in \operatorname{Cog}(Q)$. Again referring to $(a)$ we obtain a morphism $\gamma: Q \rightarrow Q^{\Lambda}$ with $h=f p \gamma$ thus proving our assertion.

(2) This can be seen from the above proof. Notice that this is also proved in $[9$, Proposition 4.4], (c) $\Rightarrow(\mathrm{e})$.

(3) follows immediately from (1).

In the next proposition we dualize the properties of $\operatorname{Gen}(Q)$-projective modules (e.g., [16, 3.3]).

$2.4 \operatorname{Cog}(Q)$-injective objects. If $Q$ is a weak subgenerator in $\mathcal{C}$, the following are equivalent:

(a) $Q$ is $\operatorname{Cog}(Q)$-injective;

(b) $Q$ is self-Ext-injective; 
(c) for each $K \in \operatorname{Cog}(Q), \operatorname{Ext}_{\mathcal{C}}^{1}(K, Q)=0$ (i.e., $\operatorname{Cog}(Q) \subset{ }^{\perp_{\mathcal{C}}} Q$ ).

If $\mathcal{C}$ has a generator $G \in \operatorname{Cog}(Q)$ with $\operatorname{Ext}_{\mathcal{C}}^{2}(G, Q)=0$ (e.g., a projective generator), then (a)-(c) are equivalent to:

(d) (i) $\operatorname{Ext}_{\mathcal{C}}^{1}\left(Q^{\Lambda}, Q\right)=0$, for any set $\Lambda$;

(ii) $\operatorname{Ext}_{\mathcal{C}}^{2}(N, Q)=0$, for each $N \in \mathcal{C}$.

Proof. (a) $\Rightarrow($ b) Let $0 \rightarrow K \rightarrow L \rightarrow N \rightarrow 0$ be an exact sequence with $N \in \operatorname{Cog}(Q)$ and consider any morphism $f: K \rightarrow Q$. By assumption, every object in $\mathcal{C}$ is a factor of a subobject of some $Q^{\Lambda}$, and so there is an epimorphism $\alpha: X \rightarrow L$ with $X \in \operatorname{Cog}(Q)$. We use this to construct the commutative diagram with exact rows,

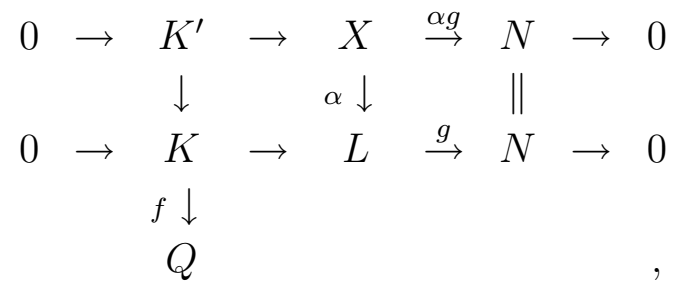

where the upper exact sequence is in $\operatorname{Cog}(Q)$ and the left hand square is a pushout. By hypothesis we can extend the diagram commutatively by some morphism $X \rightarrow Q$, and the pushout property yields the desired morphism $L \rightarrow Q$, thus proving our assertion.

$(\mathrm{b}) \Rightarrow(\mathrm{a})$ and $(\mathrm{b}) \Leftrightarrow(\mathrm{c})$ are obvious.

$(\mathrm{c}) \Rightarrow(\mathrm{d})$ Clearly $\operatorname{Ext}_{\mathcal{C}}^{1}\left(Q^{\Lambda}, Q\right)=0$, for any set $\Lambda$.

For any $N \in \mathcal{C}$, there exists an exact sequence $0 \rightarrow K \rightarrow P \rightarrow N \rightarrow 0$, where $P \in \operatorname{Cog}(Q)$ with $\operatorname{Ext}_{\mathcal{C}}^{2}(P, Q)=0$. From this we obtain the exact sequence

$$
0=\operatorname{Ext}_{\mathcal{C}}^{1}(K, Q) \rightarrow \operatorname{Ext}_{\mathcal{C}}^{2}(N, Q) \rightarrow \operatorname{Ext}_{\mathcal{C}}^{2}(P, Q)=0,
$$

proving $\operatorname{Ext}_{\mathcal{C}}^{2}(N, Q)=0$.

(d) $\Rightarrow(c)$ By the connecting morphisms of the Ext-functor (see 1.12), (ii) implies that ${ }^{\perp_{\mathcal{C}}} Q$ is closed under submodules. Hence (i) implies $\operatorname{Cog}(Q) \subset{ }^{\perp_{\mathcal{C}}} Q$.

It is easy to see that the above conditions on $Q$ imply that $Q$ is self-pseudoinjective in $\mathcal{C}$ and hence we have by 2.2 :

2.5 Corollary. Let $Q$ be a $\operatorname{Cog}(Q)$-injective weak subgenerator in $\mathcal{C}$. Then $\operatorname{Cog}(Q)$ is closed under extensions in $\mathcal{C}$, and for any $N \in \mathcal{C}, \operatorname{Hom}_{\mathcal{C}}(\operatorname{Re}(N, Q), Q)=0$.

Most of the injectivity conditions defined in 2.1 also apply for $\mathcal{C}=\sigma_{f}[Q]$ (although this is not a Grothendieck category). For example, for a self-pseudo-injective module $Q$ in $\sigma_{f}[Q], \operatorname{Cog}_{f}(Q)$ is closed under extensions in $\sigma_{f}[Q]$. With the proof of 2.3 we obtain: 


\section{$2.6 \mathrm{w}-\Pi_{f}$-quasi-injective objects.}

(1) For any $Q \in \mathcal{C}$ the following are equivalent:

(a) $Q$ is $w$ - $\Pi_{f}$-quasi-injective;

(b) $\operatorname{Hom}_{\mathcal{C}}(-, Q)$ respects exact sequences $0 \rightarrow K \rightarrow L \rightarrow N \rightarrow 0$, where $N \in \operatorname{Cog}_{f}(Q)$ and $L \in \operatorname{cop}(Q)$.

(2) If $\operatorname{cog}(Q)=\operatorname{cop}(Q)$ the following are equivalent:

(a) $Q$ is $w$-ח $\Pi_{f}$-quasi-injective;

(b) $\operatorname{Hom}_{\mathcal{C}}(-, Q)$ respects exact sequences $0 \rightarrow K \rightarrow L \rightarrow N \rightarrow 0$, where $N \in \operatorname{Cog}_{f}(Q)$ and $L \in \operatorname{cog}(Q)$.

Notice that every module in $\sigma_{f}[Q]$ is a subfactor of some $Q^{k}$. Hence a slight modification of the proof of 2.4 yields:

$2.7 \operatorname{cog}(Q)$-injective objects. For $Q \in \mathcal{C}$ the following are equivalent:

(a) $Q$ is $\operatorname{cog}(Q)$-injective;

(b) $\operatorname{Hom}_{\mathcal{C}}(-, Q)$ respects exact sequences $0 \rightarrow K \rightarrow L \rightarrow N \rightarrow 0$ in $\sigma_{f}[Q]$, where $N \in \operatorname{Cog}_{f}(Q)$;

(c) for each $K \in \operatorname{Cog}_{f}(Q), \operatorname{Ext}_{\sigma_{f}[Q]}^{1}(K, Q)=0$ (i.e., $\operatorname{Cog}_{f}(Q) \subset{ }^{\perp_{\sigma_{f}[Q]} Q}$ ).

2.8 Corollary. Let $Q \in \mathcal{C}$ be $\operatorname{cog}(Q)$-injective. Then $\operatorname{Cog}_{f}(Q)$ is closed under extensions in $\sigma_{f}[Q]$, and for any $N \in \sigma_{f}[Q], \operatorname{Hom}_{\mathcal{C}}(\operatorname{Re}(N, Q), Q)=0$.

\section{Cotilting objects}

Dualizing the definitions of tilting and self-tilting modules given in [16] leads to the following notions.

3.1 Definitions. We call $Q \in \mathcal{C}$ a cotilting object in $\mathcal{C}$ if

(i) $Q$ is $\operatorname{Cog}(Q)$-injective,

(ii) every $Q$-cogenerated module in $\mathcal{C}$ is $Q$-presented (i.e., $\operatorname{Cog}(Q)=\operatorname{Cop}(Q)$ ),

(iii) $Q$ is a weak subgenerator of $\mathcal{C}$ (i.e., $\pi[Q]=\mathcal{C}$ ).

$Q$ is called self-cotilting if it is cotilting in $\pi[Q]$, i.e., if (i) and (ii) hold in the category $\pi[Q]$. 
For a cotilting module $Q$ in $\mathcal{C}$ we have $\mathcal{C}=\pi[Q]$ (by (iii)) and so essentially it suffices to investigate self-cotilting objects in detail.

It is obvious that every injective cogenerator is cotilting in $\mathcal{C}$ and (dual to the situation for tilting modules) we have a close connection between cotilting objects and injective cogenerators (see 3.4).

$3.2 Q$ cotilting in $\sigma[Q]$. For any $Q \in \mathcal{C}$ we may ask when $Q$ is cotilting in $\sigma[Q]$. This is clearly the case when $Q$ is an injective cogenerator in $\sigma[Q]$. Notice that in general this does not imply that $Q$ is cotilting in $\pi[Q]$ (i.e., self-cotilting) even when $Q$ is a weak subgenerator in $\mathcal{C}$.

For this consider the Prüfer group $\mathbb{Z}_{p^{\infty}}$, for any prime number $p$. This is an injective cogenerator in $\sigma\left[\mathbb{Z}_{p^{\infty}}\right]$ (abelian $p$-groups) and hence is cotilting in $\sigma\left[\mathbb{Z}_{p^{\infty}}\right]$. Moreover, as a faithful $\mathbb{Z}$-module, $\mathbb{Z}_{p^{\infty}}$ is a weak subgenerator (i.e., $\pi\left[\mathbb{Z}_{p^{\infty}}\right]=$ $\mathbb{Z}$-Mod). However, $\mathbb{Z}_{p^{\infty}}$ is not cotilting in $\mathbb{Z}$-Mod by 3.4 , since it is injective but not a cogenerator in $\mathbb{Z}$-Mod.

Dualizing the characterizations of self-tilting modules and their proofs (see [16, 4.2]) we obtain characterizations of self-cotilting objects.

3.3 Self-cotilting objects. For any $Q \in \mathcal{C}$ the following are equivalent:

(a) $Q$ is self-cotilting;

(b) $\operatorname{Cog}(Q)=\operatorname{Cop}(Q)$ and $Q$ is w-П-quasi-injective ;

(c) $\operatorname{Cog}(Q)=\operatorname{Cop}(Q)$ and $Q$ is self-Ext-injective in $\pi[Q]$;

(d) $\operatorname{Cog}(Q)={ }^{\perp_{Q}} Q$;

(e) $Q$ is $\operatorname{Cog}(Q)$-injective, ${ }^{\perp_{Q}} Q$ is closed under submodules, and

(i) for $N \in \pi[Q], \operatorname{Hom}_{\mathcal{C}}(N, Q)=0=\operatorname{Ext}_{\pi[Q]}^{1}(N, Q)$ implies $N=0$, or

(ii) for any injective object (some injective cogenerator) $W \in \pi[Q]$, there exists an exact sequence

$$
0 \rightarrow Q^{\prime} \rightarrow Q^{\prime \prime} \rightarrow W \rightarrow 0, \text { where } Q^{\prime}, Q^{\prime \prime} \in \operatorname{Prod}(Q)
$$

Proof. $(\mathrm{a}) \Leftrightarrow(\mathrm{b})$ and $(\mathrm{b}) \Leftrightarrow(\mathrm{c})$ follow from 2.3 and 2.4 , respectively.

$(\mathrm{c}) \Rightarrow(\mathrm{d})$ and $(\mathrm{c}) \Rightarrow(\mathrm{e})(\mathrm{ii})$ : Consider an exact sequence $0 \rightarrow K \rightarrow L \rightarrow N \rightarrow 0$, where $L$ is a submodule of some $Q^{\Lambda}$. With a $Q$-corepresentation of $K$ (first column, 
$X \in \operatorname{Cog}(Q))$ and a pushout construction we obtain the commutative exact diagram

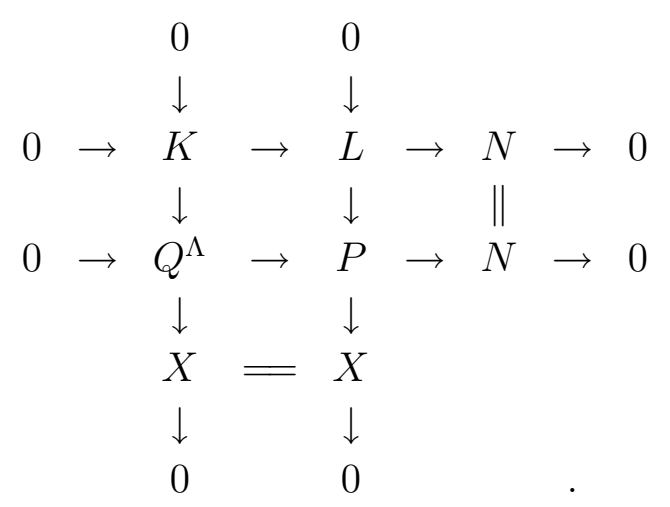

Assume $N \in{ }^{\perp_{Q}} Q$. By 2.5, $\operatorname{Cog}(Q)$ is closed under extensions in $\pi[Q]$ and hence $P \in \operatorname{Cog}(Q)$. Since $\operatorname{Ext}_{\pi[Q]}^{1}\left(N, Q^{\Lambda}\right)=0$ the central sequence splits and so $N \in$ $\operatorname{Cog}(Q)$. This proves $(\mathrm{d})$.

Now assume that $N$ is injective in $\pi[Q]$. Then, for any $Y \in \operatorname{Cog}(Q)$, we have the exact sequence

$$
0=\operatorname{Ext}_{\pi[Q]}^{1}\left(Y, Q^{\Lambda}\right) \rightarrow \operatorname{Ext}_{\pi[Q]}^{1}(Y, P) \rightarrow \operatorname{Ext}_{\pi[Q]}^{1}(Y, N)=0,
$$

and hence $\operatorname{Ext}_{\pi[Q]}^{1}(Y, P)=0$. By $(\mathrm{c})$, there exists a copresentation of $P$,

$$
0 \rightarrow P \rightarrow Q^{\Omega} \rightarrow Y \rightarrow 0, \text { where } Y \in \operatorname{Cog}(Q) .
$$

Since $\operatorname{Ext}_{\pi[Q]}^{1}(Y, P)=0$ this sequence splits and hence $P \in \operatorname{Prod}(Q)$, thus proving (e)(ii).

(e): (ii) $\Rightarrow$ (i) Let $W$ be any injective cogenerator in $\pi[Q]$. The given sequence yields the exact sequence

$$
\operatorname{Hom}_{\mathcal{C}}\left(N, Q^{\prime \prime}\right) \rightarrow \operatorname{Hom}_{\mathcal{C}}(N, W) \rightarrow \operatorname{Ext}_{\pi[Q]}^{1}(N, Q)=0,
$$

and $\operatorname{Hom}_{\mathcal{C}}(N, Q)=0$ implies $\operatorname{Hom}_{\mathcal{C}}\left(N, Q^{\prime \prime}\right)=0$ and hence $\operatorname{Hom}_{\mathcal{C}}(N, W)=0$, which means $N=0$.

(d) $\Rightarrow(\mathrm{c})$ Let $N \in \operatorname{Cog}(Q)$ and $\Lambda:=\operatorname{Hom}_{\mathcal{C}}(N, Q)$. With the canonical sequence on the top and any extension with $X \in \pi[Q]$ on the bottom we have the diagram

$$
\begin{aligned}
& 0 \rightarrow N \rightarrow Q^{\Lambda} \rightarrow L \rightarrow 0 \\
& \beta \vdots \quad \alpha \stackrel{\vdots}{v} \quad \| \\
& 0 \rightarrow Q \rightarrow X \rightarrow L \rightarrow 0
\end{aligned}
$$

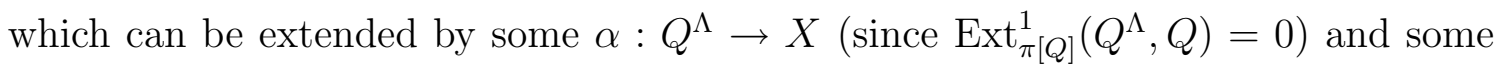
$\beta: N \rightarrow Q$ commutatively. Since $Q$ is injective with respect to the upper sequence 
we conclude (by the Homotopy Lemma) that the bottom sequence splits. Hence $\operatorname{Ext}_{\pi[Q]}^{1}(L, Q)=0$ implying $L \in{ }^{\perp_{Q}} Q=\operatorname{Cog}(Q)$ and therefore $N \in \operatorname{Cop}(Q)$.

(e)(i) $\Rightarrow(\mathrm{d})$ Let $N \in{ }^{\perp_{Q}} Q$. Then $\operatorname{Re}(N, Q) \in{ }^{\perp_{Q}} Q$ and $\operatorname{Hom}_{\mathcal{C}}(\operatorname{Re}(N, Q), Q)=0$ by 2.5. Now (i) implies $\operatorname{Re}(N, Q)=0$ which means $N \in \operatorname{Cog}(Q)$.

3.4 Corollary. For any self-cotilting module $Q \in \mathcal{C}$, the following are equivalent:

(a) $Q$ is a cogenerator in $\pi[Q]$;

(b) $Q$ is injective in $\pi[Q]$.

Proof. $(\mathrm{a}) \Rightarrow(\mathrm{b})$ is obvious.

(b) $\Rightarrow$ (a) follows from $3.3(d)$, since for $Q$ injective in $\pi[Q]$ clearly ${ }^{{ }_{Q}} Q=\pi[Q]$.

To make a self-cotilting object $Q \in \mathcal{C}$ cotilting in $\mathcal{C}$ some condition is needed to turn $Q$ into a weak subgenerator in $\mathcal{C}$.

3.5 Cotilting objects. For any $Q \in \mathcal{C}$ the following are equivalent:

(a) $Q$ is cotilting in $\mathcal{C}$;

(b) $Q$ is self-cotilting and a weak subgenerator in $\mathcal{C}$;

If $\mathcal{C}$ has a projective (sub-) generator then (a)-(b) are equivalent to:

(c) $\operatorname{Cog}(Q)={ }^{\perp_{\mathcal{C}}} Q$;

(d) (i) $\operatorname{Ext}_{\mathcal{C}}^{1}\left(Q^{\Lambda}, Q\right)=0$, for any set $\Lambda$;

(ii) $\operatorname{Ext}_{\mathcal{C}}^{2}(N, Q)=0$, for each $N \in \mathcal{C}$;

(iii) for $N \in \pi[Q], \operatorname{Hom}_{\mathcal{C}}(N, Q)=0=\operatorname{Ext}_{\mathcal{C}}^{1}(N, Q)$ implies $N=0$;

(e) (i) and (ii) as in (e) and

(iv) for some injective cogenerator $W \in \mathcal{C}$, there exists an exact sequence

$$
0 \rightarrow Q^{\prime} \rightarrow Q^{\prime \prime} \rightarrow W \rightarrow 0, \text { where } Q^{\prime}, Q^{\prime \prime} \in \operatorname{Prod}(Q)
$$

Proof. The assertions follow by 3.3, 2.4, and the observation that any projective object of $\mathcal{C}$ belongs to ${ }^{\perp_{\mathcal{C}}} Q$. The latter implies that in (c),(d) and (e), $Q$ is a weak subgenerator in $\mathcal{C}$.

For any $R$-module $Q, \pi[Q]=R / \operatorname{An}(Q)$-Mod and hence it has a projective generator. So we obtain from 3.3 and 3.5:

3.6 Self-cotilting modules. For any left $R$-module $Q$, put $\bar{R}=R / \operatorname{An}(Q)$. Then the following are equivalent: 
(a) $Q$ is self-cotilting;

(b) $\operatorname{Cog}(Q)=\operatorname{Cop}(Q)$ and $Q$ is w-П-quasi-injective ;

(c) $Q$ is cotilting in $\bar{R}$-Mod;

(d) $\operatorname{Cog}(Q)={ }^{\perp_{\bar{R}}} Q$;

(e) (i) $\operatorname{Ext}_{\frac{1}{R}}\left(Q^{\Lambda}, Q\right)=0$, for any set $\Lambda$;

(ii) $\operatorname{Ext}_{\bar{R}}(N, Q)=0$, for each $N \in \bar{R}$-Mod;

(iii) for $N \in \bar{R}$-Mod, $\operatorname{Hom}_{R}(N, Q)=0=\operatorname{Ext}_{\bar{R}}(N, Q)$ implies $N=0$;

(f) (i), (ii) as in (e) and

(iv) for some injective cogenerator $W \in \bar{R}$-Mod, there exists an exact sequence

$$
0 \rightarrow Q^{\prime} \rightarrow Q^{\prime \prime} \rightarrow W \rightarrow 0, \text { where } Q^{\prime}, Q^{\prime \prime} \in \operatorname{Prod}(Q)
$$

3.7 Cotilting in $R$-Mod. By definition, $Q$ is cotilting in $R$-Mod if and only if it is self-cotilting and faithful. Hence 3.6 yields characterizations of these modules by replacing $\bar{R}$ by $R$ in (c),(d),(e) and (f).

Notice that for this case $(d) \Leftrightarrow(e)$ was proved in Colpi-D'Este-Tonolo [7, Proposition 1.7] and $(d)$ implies $\operatorname{Cog}(Q)=\operatorname{Cop}(Q)$ is shown in [7, Proposition 1.8]. Moreover $(d) \Rightarrow(f)$ corresponds to Angeleri-Hügel-Tonolo-Trlifaj [3, Proposition 2.3].

From 3.5 we obtain the

3.8 Corollary. For $Q$ cotilting in $R$-Mod, the following are equivalent:

(a) $Q$ is a cogenerator in $R$-Mod;

(b) $Q$ is injective in $R$-Mod.

To investigate dualities we introduce a finite version of cotilting objects.

3.9 Definition. We call $Q \in \mathcal{C}$ an $f$-cotilting object if

(i) $Q$ is $\operatorname{cog}(Q)$-injective,

(ii) every finitely $Q$-cogenerated object in $\mathcal{C}$ is finitely $Q$-copresented (i.e., $\operatorname{cog}(Q)=\operatorname{cop}(Q))$.

For example, every semisimple $R$-module is f-cotilting.

Remark. In Angeleri-Hügel-Valenta [2] finitely cotilting modules $Q$ are defined as "cotilting" modules (with a slightly different definition) which are finitely generated $R$-modules such that $\operatorname{Hom}_{R}(X, Q)$ is a finitely generated $\operatorname{End}_{R}(Q)$-module, for any 
finitely generated $R$-module $X$. It is easy to see that over noetherian rings such modules are f-cotilting in the sense defined above (compare [2, Corollary 5.2]).

Recall that an object $Q$ is injective in $\sigma_{f}[Q]$ if and only if it is injective in $\sigma[Q]$ (i.e., $Q$-injective) and $Q$ is an injective cogenerator in $\sigma_{f}[Q]$ if and only if it is an injective cogenerator in $\sigma[Q]$. Similar to 3.4 we have now:

3.10 Proposition. For any f-cotilting object $Q \in \mathcal{C}$, the following are equivalent:

(a) $Q$ is a cogenerator in $\sigma_{f}[Q]$ (in $\left.\sigma[Q]\right)$;

(b) $Q$ is injective in $\sigma_{f}[Q]$ (in $\sigma[Q]$ ).

Proof. $(a) \Rightarrow(b)$ is obvious.

$(\mathrm{b}) \Rightarrow(\mathrm{a})$ (compare proof of $3.3,(\mathrm{c}) \Rightarrow(\mathrm{d})$ ) For any subobject $K \subset Q$ we obtain the commutative exact diagram, where the first column is a $Q$-copresentation of $K$ $(k \in \mathbb{N}, X \in \operatorname{Cog}(Q))$, by a pushout construction

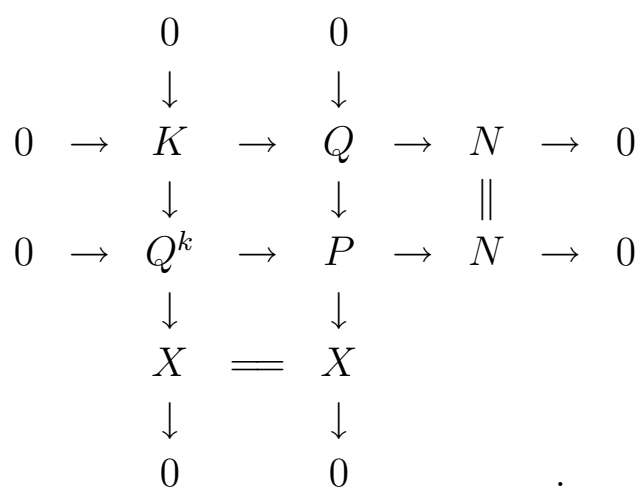

Since $P$ is in $\sigma[Q]$ and $Q$ is $Q$-injective, the central column splits implying that $P \in \operatorname{Cog}(Q)$. For the same reasons the central row splits and hence $N \in \operatorname{Cog}(Q)$. From this we conclude that $Q$ is a cogenerator in $\sigma[Q]$ (e.g., $[14,16.5]$ ).

The condition $\operatorname{cog}(Q)=\operatorname{cop}(Q)$ holds trivially provided $Q$ is a cogenerator in $\sigma_{f}[Q]$ but it need not follow from the condition $\operatorname{Cog}(Q)=\operatorname{Cop}(Q)$. In fact it is related to some finiteness properties.

3.11 Proposition. Let $Q \in \mathcal{C}$ be $w$ - $\Pi_{f}$-quasi-injective. Then for every $K \in \operatorname{cop}(Q)$, $\operatorname{Hom}_{\mathcal{C}}(K, Q)$ is a finitely generated right $\operatorname{End}_{\mathcal{C}}(Q)$-module.

Proof. This is obvious.

Adapting the proofs of 3.3 we obtain characterizations of f-cotilting objects.

3.12 f-cotilting objects. For any $Q \in \mathcal{C}$ and $S=\operatorname{End}_{\mathcal{C}}(Q)$, the following are equivalent: 
(a) $Q$ is f-cotilting;

(b) $\operatorname{cog}(Q)=\operatorname{cop}(Q)$ and $Q$ is $w$ - $\Pi_{f}$-quasi-injective;

(c) $\operatorname{cog}(Q)=\operatorname{cop}(Q)$ and $\operatorname{Cog}_{f}(Q) \subset^{\perp_{f}} Q$;

(d) $\operatorname{Cog}_{f}(Q)={ }^{\perp_{f}} Q$, and for every $K \in \operatorname{cog}(Q), \operatorname{Hom}_{\mathcal{C}}(K, Q) \in \bmod -S$;

(e) (i) $Q$ is $\operatorname{cog}(Q)$-injective, ${ }^{{ }_{f}} Q$ is closed under submodules,

(ii) for $N \in \sigma_{f}[Q], \operatorname{Hom}_{\mathcal{C}}(N, Q)=0=\operatorname{Ext}_{\sigma_{f}[Q]}^{1}(N, Q)$ implies $N=0$, and

(iii) for every $K \in \operatorname{cog}(Q), \operatorname{Hom}_{\mathcal{C}}(K, Q) \in \bmod -S$.

Proof. $(\mathrm{a}) \Leftrightarrow(\mathrm{b})$ and $(\mathrm{b}) \Leftrightarrow(\mathrm{c})$ follow from 2.6 and 2.7 , respectively.

(c) $\Rightarrow(\mathrm{d})$ In the proof of 3.3, (c) $\Rightarrow(\mathrm{d})$, take $N \in^{\perp_{f}} Q$ and $\Lambda$ a finite index set.

$(\mathrm{d}) \Rightarrow(\mathrm{c})$ Let $N \in \operatorname{cog}(Q)$ and $f_{1}, \ldots, f_{k}$ a generating set for the $S$-module $\operatorname{Hom}_{\mathcal{C}}(K, Q)$. The $f_{i}$ 's yield a canonical monomorphism $N \rightarrow Q^{k}$. Now procede as in the proof of $3.3,(\mathrm{~d}) \Rightarrow(\mathrm{c})$.

The remaining implications can also be transferred from 3.3.

More examples of f-cotilting objects will be given at the end of the paper.

\section{Reflexive modules and dualities}

To avoid technical complications we restrict our study of dualities to module categories. We investigate dualities induced by any left $R$-module $Q$ with $S=\operatorname{End}_{R}(Q)$.

4.1 Canonical functors. Related to ${ }_{R} Q_{S}$ we have the adjoint pair of functors

$$
D: \text { R-Mod } \stackrel{\operatorname{Hom}_{R}(-, Q)}{\longrightarrow} \text { Mod-S, } D^{\prime}: \operatorname{Mod}-\mathrm{S} \stackrel{\operatorname{Hom}_{S}(-, Q)}{\longrightarrow} \text { R-Mod, }
$$

and for any $N \in \mathrm{R}$-Mod and $X \in \mathrm{S}-\mathrm{Mod}$, the canonical (evaluation) morphisms

$$
\begin{array}{ll}
\Phi_{N}: N \rightarrow D^{\prime} D(N), & n \mapsto[\beta \mapsto(n) \beta], \\
\Phi_{X}^{\prime}: X \rightarrow D D^{\prime}(X), \quad x \mapsto[\alpha \mapsto \alpha(x)] .
\end{array}
$$

where

$$
\operatorname{Ke} \Phi_{N}=\operatorname{Re}(N, Q), \quad \operatorname{Ke} \Phi_{X}^{\prime}=\operatorname{Re}(N, Q) .
$$

4.2 (Semi-)reflexive modules. A module $N \in R$-Mod is called (semi-) $Q$-reflexive if $\Phi_{N}: N \rightarrow D^{\prime} D(N)$ is an isomorphism (epimorphism). Similarly (semi-) $Q$ reflexive objects in Mod-S are defined. It is straightforward to prove:

$N \in \sigma[Q]$ is semi-Q-reflexive if and only if $N / \operatorname{Re}(N, Q)$ is $Q$-reflexive.

The class of all $Q$-reflexive modules in R-Mod (in Mod-S) is denoted my $\operatorname{Ref}_{R}(Q)$ (resp., $\left.\operatorname{Ref}_{S}(Q)\right)$. 
Obviously we have the following

4.3 Basic duality. For any $R$-module $Q$, the functor

$$
D=\operatorname{Hom}_{R}(-, Q): \operatorname{Ref}_{R}(Q) \rightarrow \operatorname{Ref}_{S}(Q)
$$

defines a duality with inverse $D^{\prime}=\operatorname{Hom}_{S}(-, Q)$.

For any $N \in$ R-Mod consider an exact sequence $S^{\left(\Lambda^{\prime}\right)} \rightarrow S^{(\Lambda)} \rightarrow D(N) \rightarrow 0$ in Mod-S. By left exactness of $\operatorname{Hom}_{S}(-, Q)$ we obtain the exact sequence

$$
0 \rightarrow \operatorname{Hom}_{S}(D(N), Q) \rightarrow Q^{\Lambda} \rightarrow Q^{\Lambda^{\prime}}
$$

Now if $N \in \operatorname{Ref}_{R}(Q)$, i.e., $N \simeq D^{\prime} D(N)$, we conclude $N \in \operatorname{Cop}_{R}(Q)$.

4.4 Classes of modules related to $Q$. We have the following inclusions:

(1) $D^{\prime}(\operatorname{Mod}-\mathrm{S}) \subset \operatorname{Cop}_{R}(Q), \quad D(\mathrm{R}-\mathrm{Mod}) \subset \operatorname{Cop}_{S}(Q)$.

(2) $\operatorname{add}(Q) \subset \operatorname{Ref}_{R}(Q) \subset \operatorname{Cop}_{R}(Q) \subset \operatorname{Cog}_{R}(Q) \subset \mathrm{R}-\operatorname{Mod}$.

(3) $\operatorname{add}(Q) \subset \operatorname{cop}_{R}(Q) \subset \operatorname{cog}_{R}(Q) \subset \sigma_{f}[Q]$.

We investigate the properties of the classes considered above in view of certain injectivity conditions.

A submodule $K \subset M$ in called $Q$-closed in $M$ provided $M / K \in \operatorname{Cog}(Q)$.

4.5 w-П-quasi-injective modules. Let ${ }_{R} Q$ be $w$-П-quasi-injective. Then:

(1) $\operatorname{Ref}_{R}(Q)$ is closed under $Q$-closed submodules; in particular, $\operatorname{Ref}_{R}(Q)$ is closed under kernels.

(2) Every factor module of a $Q$-reflexive right $S$-module is semi-Q-reflexive.

Proof. (1) Let $P \in \operatorname{Ref}_{R}(Q)$ with a $Q$-closed submodule $K \subset P$. Then we have an exact commutative diagram,

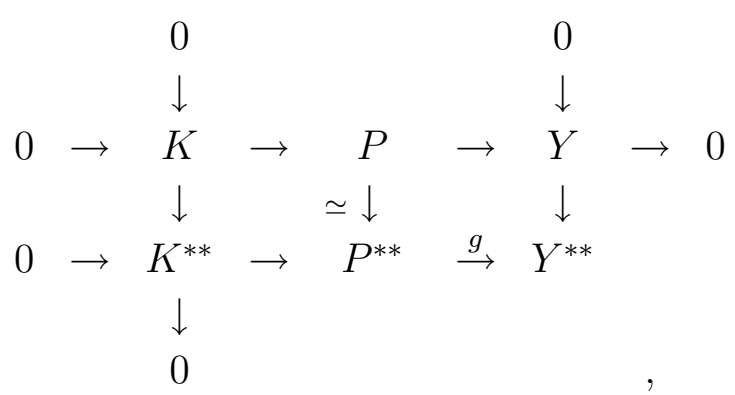

where $Y \in \operatorname{Cog}(Q)$. This shows that $K$ is $Q$-reflexive. 
(2) Let $X \in \operatorname{Ref}_{S}(Q)$. For any epimorphism $X \rightarrow L$ we have the exact commutative diagram

$$
\begin{array}{ccccc}
X & \rightarrow & L & \rightarrow & 0 \\
\simeq \downarrow & & \downarrow & & \\
X^{* *} & \rightarrow & L^{* *} & \rightarrow & 0,
\end{array}
$$

which shows that $L$ is semi- $Q$-reflexive.

The question arises to which extent properties of $\operatorname{Ref}_{R}(Q)$ imply injectivity conditions on $Q$. Since $\operatorname{Ref}_{R}(Q)$ is not closed under products we are not able to get a general assertion converse to 4.5. However the situation is different if we restrict our considerations to finite products as we will see below. First we formulate a finite version of 4.5. Since for any $k \in \mathbb{N}, Q^{k} \in \operatorname{Ref}_{R}(Q)$ and $S^{k} \in \operatorname{Ref}_{S}(Q)$, essentially the same proof yields:

$4.6 \mathrm{w}-\Pi_{f}$-quasi-injective modules. Let $Q$ be a $w$ - $\Pi_{f}$-quasi-injective $R$-module. Then:

(1) $\operatorname{cop}(Q) \subset \operatorname{Ref}_{R}(Q)$.

(2) Every finitely generated right $S$-module is semi-Q-reflexive.

(3) By restriction we have the functor $\operatorname{Hom}_{R}(-, Q): \operatorname{cop}(Q) \rightarrow \bmod -S$.

4.7 Proposition. Let $Q \in R$-Mod. Assume $\operatorname{cop}(Q) \subset \operatorname{Ref}_{R}(Q)$ and that all finitely generated right $S$-modules are semi-Q-reflexive. Then $Q$ is $w$ - $\Pi_{f}$-quasi-injective.

Proof. (compare $[14,47.12]$ ) We have to show that $\operatorname{Hom}_{R}(-, Q)=(-)^{*}$ is exact on sequences

$$
0 \rightarrow K \stackrel{f}{\rightarrow} Q^{k} \rightarrow Y \rightarrow 0, \text { where } Y \in \operatorname{Cog}(Q) .
$$

From the inclusion $\delta: \operatorname{Im} f^{*} \rightarrow K^{*}$ we obtain the commutative diagram with exact rows

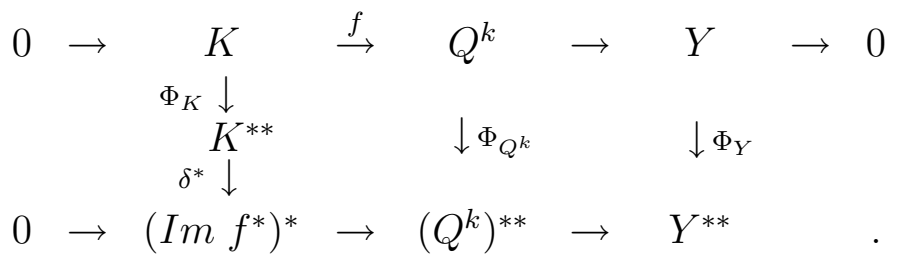

Since $\Phi_{Q^{k}}$ is an isomorphism and $\Phi_{Y}$ is a monomorphism, $\Phi_{K} \delta^{*}$ is an isomorphism. Now $K$ being $Q$-reflexive implies that $\delta^{*}$ is also an isomorphism. We have the commutative diagram

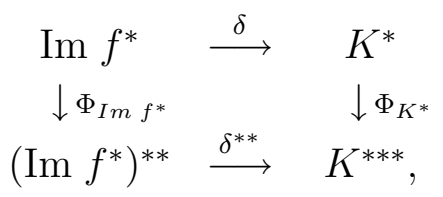


where $\Phi_{I m f^{*}}$ is surjective (by our assumptions) and $\delta^{* *}$ is an isomorphism. This clearly implies that $\delta$ is an isomorphism which means that $(-)^{*}$ is exact on the given sequence.

Combining the results just derived we obtain:

4.8 Injectivity and duality. For $Q \in R$-Mod the following are equivalent:

(a) $Q$ is $w$-П $\Pi_{f}$-quasi-injective;

(b) $\operatorname{cop}(Q) \subset \operatorname{Ref}_{R}(Q)$ and all finitely generated right $S$-modules are semi-Qreflexive;

(c) $\operatorname{cop}(Q) \subset \operatorname{Ref}_{R}(Q)$ and $\bmod -S \cap \operatorname{Cog}_{S}(Q) \subset \operatorname{Ref}_{S}(Q)$;

(d) $\operatorname{Hom}_{R}(-, Q): \operatorname{cop}(Q) \rightarrow \bmod -S \cap \operatorname{Cog}_{S}(Q)$ is a duality.

Proof. $(a) \Rightarrow(b)$ follows from 4.6.

$(b) \Leftrightarrow(c)$ is clear by the comments in 4.2 .

$(c) \Rightarrow(a)$ holds by Proposition 4.7 .

$(c) \Leftrightarrow(d)$ is obvious.

4.9 Remark. Dualizing the notion of s- $\sum$-quasi-projective used in Sato [10] we may call an $R$-module $Q s$-П-quasi-injective if $\operatorname{Hom}_{R}(-, Q)$ is exact on sequences

$$
0 \rightarrow K \rightarrow Q^{\Lambda} \rightarrow Q^{\Lambda^{\prime}} \text {, for any (or finite) sets } \Lambda, \Lambda^{\prime} .
$$

With similar proofs one gets that for such modules, $\operatorname{Hom}_{R}(-, Q)$ induces an equivalence between the kernels of morphisms $Q^{k} \rightarrow Q^{l}, k, l \in \mathbb{N}$ (a subclass of $\operatorname{cog}(Q)$ ) and the finitely presented right $S$-modules (compare $[17,4.6]$ ).

By the definition of f-cotilting modules, 4.8 yields immediately:

4.10 f-cotilting and duality. For $Q \in R$-Mod the following are equivalent:

(a) $Q$ is f-cotilting;

(b) $\operatorname{cog}(Q) \subset \operatorname{Ref}_{R}(Q)$ and all finitely generated right $S$-modules are semi-Qreflexive;

(c) $\operatorname{cog}(Q) \subset \operatorname{Ref}_{R}(Q)$ and mod- $S \cap \operatorname{Cog}_{S}(Q) \subset \operatorname{Ref}_{S}(Q)$;

(d) $\operatorname{Hom}_{R}(-, Q): \operatorname{cog}(Q) \rightarrow \bmod -S \cap \operatorname{Cog}_{S}(Q)$ is a duality. 
Finally we mention some more examples of f-cotilting objects. Clearly an object $Q$ which is an injective cogenerator in $\mathcal{C}$ - or more generally in $\sigma[Q]$ - is f-cotilting. In particular any semisimple object has this property.

Cotilting modules $Q$ are f-cotilting, provided for every $K \in \operatorname{cog}(Q), \operatorname{Hom}_{\mathcal{C}}(K, Q) \in$ mod- $\operatorname{End}_{\mathcal{C}}(Q)$. Sufficient for the latter condition is that $Q$ is noetherian both as left $R$ - and right $\operatorname{End}_{\mathcal{C}}(Q)$-module. This situation was considered in Wang-Xu [13, Theorem 2 and 3]. If ${ }_{R} Q$ is cotilting and artinian the conditions are also satisfied and this is the situation usually considered in representation theory.

It was already mentioned in the remark following 3.9 that over noetherian rings the "finitely cotilting" modules studied in Angeleri-Hügel-Valenta [2] are f-cotilting modules in our sense. Notice that artinian cotilting modules need not be "finitely cotilting" in the sense of [2] since they need not be finitely generated as modules over their endomorphism ring (see Example 2.3 in [1]).

The Morita duality as described in $[14,47.12]$ is a special case of 4.10 . Notice that in $[14,47.12]$ the class of reflexive $R$-modules is closed under factor modules and submodules while in 4.10 this class is only closed under submodules.

\section{References}

[1] L. Angeleri-Hügel, Finitely cotilting modules, Comm. Algebra 28(4) (2000), $2147-2172$.

[2] L. Angeleri-HüGel, H. VAlenta, A duality result for almost split sequences, Coll. Math. 80 (1999), 267-292.

[3] L. Angeleri-Hügel, A. Tonolo, J. TrlifaJ, Tilting preenvelopes and cotilting precovers, Algebras and Repres. Theory, to appear

[4] R.R. Colby, A generalization of Morita duality and the tilting theorem, Comm. Algebra 17(7) (1989), 1709-1722.

[5] R. ColpI, Cotilting bimodules and their dualities, Interactions Between Ring Theory and Representations of Algebras, F. van Oystaeyen, M. Saorin (ed), Marcel Dekker (2000), 81-93.

[6] R. Colpi, K.R. Fuller, Cotilting modules and bimodules, Pac. J. Math. 192(2) (2000), 275-291.

[7] R. Colpi, G. D'Este And A. Tonolo, Quasi-tilting modules and counter equivalences, J. Algebra 191 (1997), 461-494.

[8] R. Colpi, A. Tonolo and J. TrlifaJ, Partial cotilting modules and the lattices induced by them, Comm. Algebra 25 (1997), 3225-3237. 
[9] J. Rada, M. SaOrín, A. Del VAlle, Reflective and coreflective subcategories, Glasgow Math. J. 42(1) (2000), 97-113.

[10] M. Sato, On equivalences between module categories, J. Algebra 59 (1979), 412-420.

[11] B. Stenström, Rings of Quotients, Springer-Verlag, Berlin (1975).

[12] T. Wakamatsu, Pseudo-projectives and pseudo-injectives in Abelian categories, Math. Rep. Toyama Univ. 2 (1979), 133-142.

[13] Wang Mingyi, Xu Xonghua, *-modules, co-*-modules and cotilting modules over Noetherian rings, Science in China (Series A) 39(1) (1996), 48-55.

[14] R. Wisbauer, Foundations of Module and Ring Theory, Gordon and Breach, Reading (1991).

[15] R. Wisbauer, On module classes closed under extensions, Rings and radicals, Gardner, Liu Shaoxue, Wiegandt (ed.), Pitman RN 346 (1996), 73-97.

[16] R. Wisbauer, Tilting in module categories, Abelian groups, module theory, and toplogy, Dikranjan, Salce (ed.), Marcel Dekker LNPAM 201 (1998), 421444.

[17] R. Wisbauer, Static modules and equivalences, Interactions Between Ring Theory and Representations of Algebras, F. van Oystaeyen, M. Saorin (ed), Marcel Dekker (2000), 423-449.

[18] Huang Zhaoyong, On a generalization of the Auslander-Bridger transpose, Comm. Algebra 27(12) (1999), 5791-5812. 